\title{
Índice estandarizado de precipitación (SPI) para la caracterización de sequías meteorológicas en la cuenca del río Dagua-Colombia ${ }^{1}$
}

\section{Standardized precipitation index (SPI) for meteorological drought characterization in the Dagua river basin-Colombia}

\author{
Wilmar Loaiza Cerón*, Yesid Carvajal Escobar ${ }^{* *}$ y \\ Olga Lucia Baquero Montoya***
}

\section{INTRODUCCIÓN}

Históricamente, la sequía ha sido uno de los «desastres» de origen hidrometeorológico que más afecta la actividad agrícola, el bienestar y la soberanía alimentaria de la sociedad. Las comunidades ancestrales adquirieron conciencia de los estrechos vínculos entre los cultivos y las condiciones meteorológicas; implementando medidas de adaptación para buscar cultivos alimentarios alternativos. Sin embargo, un solo año agrícola marcado por una intensa sequía se traducía en la reducción de la producción en las cosechas, que general-

\footnotetext{
${ }^{1}$ Los autores agradecen al Programa de Jóvenes Investigadores e Innovadores del Departamento Administrativo de Ciencia, Tecnología e Innovación COLCIENCIAS, a la Maestría en Desarrollo Sustentable y al grupo IREHISA de la Universidad del Valle financiado por el programa UNESCO-IHE Partnership Research Fund (UPaRF) y al Proyecto Estrategias agroecológicas para la sostenibilidad y adaptación a la variabilidad climática y el cambio climático en la cuenca alta del río Dagua, financiado por la Universidad del Valle.

* Universidad del Valle, Centro Internacional de Agricultura Tropical-CIAT, w.loaiza@cgiar.org

** Universidad del Valle, yesid.carvajal@correounivalle.edu.co

*** Universidad del Valle, olga.baquero@correounivalle.edu.co
} 
mente impulsaban a las poblaciones a migrar hacia otras regiones. (Organización Meteorológica Mundial-OMM, 2006²; Below et al., 2007). En la actualidad, incluso las sequías débiles tienen graves repercusiones en el sector rural de los países en desarrollo (Kallis, 2008), debido a que el 75\% de los pobres del mundo habitan en zonas rurales y dependen de la agricultura para su subsistencia (Carvajal et al., 2008); ésta tendencia continuará a la par con el incremento de la población mundial y las necesidades de producción alimentaria, que a su vez aumentarán en un 70\% para el 2050 (Rahman \& Westley, 2002; Dasgupta, 2004³).

Ante la preocupación mundial por la variabilidad (VC) y el cambio climático (CC) como la mayor amenaza ambiental de los últimos tiempos (Vincent, 2007; Brown \& Funk, 2008), el estudio de las sequías cobra mayor importancia. Definir el fenómeno es el primer paso en un análisis riguroso del mismo; se han realizado esfuerzos buscando precisar qué es una sequía en el sentido conceptual y numérico del tema, (Wilhite \& Buchanan, 2005; Seth, 2003; Patrick, 2003). Sin embargo, es una tarea difícil, sino imposible, debido a la complejidad antes mencionada, al sinnúmero de actividades socio-económicas involucradas y en el caso colombiano, a la compleja variabilidad de la lluvia tropical (National Drought Mitigation Center - NDMC, 2006). La OMM (2006) define la sequía como:

un fenómeno perjudicial y subrepticio que se produce a raíz de niveles de precipitación inferiores a lo esperado o a lo normal y que, cuando se prolonga durante una estación o durante períodos más largos, hace que las precipitaciones sean insuficientes para responder a las demandas sociales y del medio ambiente. La sequía es una anomalía transitoria y en ello se diferencia de la aridez, que es una característica permanente del clima.

Este déficit de precipitación puede sobrevenir en poco tiempo o tardar meses en manifestarse a través de la disminución del caudal de los ríos, el nivel de los embalses y las aguas subterráneas. El déficit de precipitación empieza a manifes-

\footnotetext{
${ }^{2}$ Organización Meteorológica Mundial (OMM) (2006): Vigilancia y alerta temprana de la sequía: conceptos, progresos y desafíos futuros. Información meteorológica y climática para el desarrollo agrícola sostenible. Suiza, OMM, No 1006, 28 pp. http://www.ub.es/geocrit/b3w-571.htm _edn7http://www.ub.es/geocrit/b3w-571.htm - _edn7http://www.ub.es/geocrit/b3w-571.htm _edn7. (Verificadas el 4/5/2015).

${ }^{3}$ Dasgupta, P. (2004): "World Poverty: Causes and Pathways, en World Bank's Annual Bank Conference on Development Economics (May 2003, Bangalore)", in: Proceedings of the World Bank Conference on Development Economics, 2003 (Supplement to the World Bank Economic Review and the World Bank Research Observer). Washington DC, World Bank, p. 44.
} 
tarse en la disminución de agua en los suelos, por lo que la agricultura suele ser el primer sector afectado (OMM, 2006). La sequía es un fenómeno climático complejo que afecta la sociedad y el medio ambiente (Valiente, 2001); los riesgos más comunes en el sector agrícola son: reducción de rendimientos en cultivos, menor desarrollo vegetativo, reducción de germinación de las plantas, de número o tamaño de los frutos, pérdidas parciales o totales de las cosechas, incremento de plagas y enfermedades en las plantas, disminución de la producción agropecuaria, y de la producción de alimentos, entre otros (Bernal, 2010 ${ }^{4}$.

En los últimos años, se evidencia un cambio de enfoque en la comunidad internacional de gestión del riesgo y adaptación en la forma de abordar los desastres, generalmente, más enfocada en la amenaza que las condiciones de la población expuesta y la construcción social del riesgo (Vargas, 2002). Recientemente, la preocupación se está orientando hacia las comunidades más afectadas, reconociéndose que la vulnerabilidad es la principal causa de las pérdidas y deben ser objeto de acciones para reducirlas, enmarcándolas en el contexto de desarrollo sostenible, como lo plantean los objetivos de desarrollo del milenio (ONU, 20005; Lavell et al., 2003; OMM, 2006; Cepal, 20106). Esto ha evidenciado la necesidad de realizar estudios orientados a identificar medidas adecuadas para mitigar sus efectos y para adaptarse mediante estrategias de Gestión y Reducción de Riesgo de Desastres en la planificación del desarrollo (Bass et al. ${ }^{7}, 2008$; Seth, 2003; Mendelsohn \& Dinar, 1999; FAO, 20058; Aldunce et al., 2009; Debels et al., 2008).

${ }^{4}$ Bernal, O. (2010): "Uso del pronóstico en el sector agropecuario", en: Congreso Nacional del Clima «El desarrollo económico de Colombia bajo un nuevo escenario climático» (2do, 2010, Bogotá Colombia). Bogotá, Colombia, Instituto de Hidrología, meteorología y Estudios Ambientales (IDEAM), p. 23.

${ }^{5}$ Organización de las Naciones Unidas - ONU. (2000): 55/2. Declaración del Milenio, Asamblea General, Quincuagésimo quinto período de sesiones Tema 60 b) del programa. Nueva York, Naciones Unidas, $10 \mathrm{pp}$.

6 CEPAL (2010): "Desastres y desarrollo: el impacto en 2010 (Cifras preliminares)", en: Unidad de Desastres, División de Desarrollo Sostenible y Asentamientos Humanos, Boletín 2, 16 de diciembre de 2010, 11 pp. [en línea]. Disponible en: http://www.cepal.org/desastres/noticias/ noticias/2/42102/desastres2010_web.pdf (Verificada: 15/09/2015).

7 Bass, S.; Ramasamy, S.; Dey, J. \& Battista, F. (2008): Disaster Risk Management Systems Analysis. Rome, environment, climate change and bioenergy division, Food and agriculture organization of the United Nations, 9 pp.

${ }^{8}$ FAO (2005): Special Event on Impact of Climate Change, Pests and Diseases on Food Security and Poverty Reduction. Background Document. Rome, $31^{\text {st }}$ Session of the Committee on World Food Security, 9 pp. 
A pesar de la importancia que esta amenaza reviste para las comunidades agrícolas de secano del país, por su vulnerabilidad, la cual se acentúa durante la ocurrencia de la fase cálida del fenómeno ENOS; se puede decir que son pocos los estudios que disponen de información para cuantificar la probabilidad de ocurrencia del fenómeno para la evaluación de su riesgo; situación ésta que agudiza el problema de la pobreza rural y la soberanía alimentaria que vive el país.

En este trabajo, se utilizó el Índice de Precipitación Estandarizado (SPI, Standardized Precipitation Index), desarrollado por Mckee et al. (1993), reconocido por su facilidad de uso a diferentes escalas temporales y porque aporta mejores resultados que otros índices para explicar los impactos de la sequía, (Guttman, 1998; Vicente \& López, 2005). Mediante el uso del SPI, se lograron evaluar diferentes parámetros de las sequías, tales como intensidad, magnitud, frecuencia, duración y cobertura espacial, resultados que se constituyen en un insumo importante para la planificación y adaptación de los sistemas productivos agrícolas de la zona.

\section{ANTECEDENTES}

La sequía es una amenaza que puede convertirse en desastre cuando se extiende a través de un prolongado período de tiempo, y genera insuficiencia de oferta hídrica para satisfacer las demandas ambientales y humanas (Wilhite $\&$ Buchanan, 2005; Seth, 2003); por lo anterior, son consideradas como el mayor peligro individual y natural (Patrick, 2003). Below et al. (2007) reportan que entre 1900 y 2004, las sequías causaron más del 50\% de las muertes por desastres naturales, representando el $35 \%$ de la población afectada y el $7 \%$ de las pérdidas económicas, después de las inundaciones y los sismos.

La variabilidad climática de Colombia está fuertemente influenciada por El Niño Oscilación de Sur (ENOS), caracterizado por un aumento de las temperaturas superficiales del Pacífico tropical que ocasiona grandes alteraciones en los patrones de circulación de vientos, presiones atmosféricas superficiales, precipitación y nubosidad sobre el océano Pacífico; la fase fría del ENOS se conoce como La Niña y la fase cálida como EL Niño (Rojo \& Carvajal, 2010).

Los episodios cálidos con mayor impacto en los diferentes sectores de la vida nacional están referidos a los períodos de 1991-1992 y 1997-1998. El costo de los daños ocasionados por El Niño de 1997-1998 en la región andina se estimó en $\$ 7.543$ millones de US, distribuidos así, en millones: Perú 
US\$3.498, Ecuador US\$2.882 millones, Colombia US\$564, Bolivia US\$527 y Venezuela US\$72 (Jovel, $\left.2000^{9}\right)$.

El estudio de Zuluaga (2009) permite asociar los eventos de sequía con la ocurrencia del Fenómeno El Niño al hacer un recuento de los episodios más intensos durante el período analizado. Para la región Andina, según el índice de precipitación estandarizado, los eventos de sequías extremas que tuvieron lugar en orden de severidad son: 1997-98, 1991-92, 1982-83, 1976-77 y 1987-88. Dichos períodos coinciden con la ocurrencia de la fase cálida del ENOS. Esta situación se repite en las demás regiones con algunas variaciones en el orden de los eventos más intensos. En el caso de la región Pacífica los valores más críticos ocurrieron en los períodos: 1984-85, 1997-98, 1991-92 y 1987-88.

La cuenca del río Dagua se encuentra localizada en el pacífico vallecaucano, al Occidente del País (figura 1) y presenta un área de $1.422 \mathrm{Km}^{2}$ aproximadamente, desde la costa del Océano Pacífico a $0 \mathrm{msnm}$ hasta los 2600 msnm en la vertiente occidental de la cordillera occidental. La temperatura está determinada por los pisos altitudinales y varía entre los $30^{\circ} \mathrm{C}$ en la parte costera, $18^{\circ} \mathrm{C}$ en la parte media y $12^{\circ} \mathrm{C}$ en la parte alta (Corporación Autónoma Regional del Valle del Cauca-CVC, $2008^{10}$ ). En su parte baja (al Oeste) presenta un comportamiento monomodal, con una precipitación total anual entre $2000 \mathrm{~mm}-8000 \mathrm{~mm}$, en contraste la zona media y alta (al Este) presenta un comportamiento bimodal con una precipitación total anual entre $800 \mathrm{~mm}-1600 \mathrm{~mm}$, caracterizado por la migración de la faja de convergencia intertropical. Esta zona es importante para Colombia, porque por ella cruza la principal vía que comunica el interior del país con el Pacífico y se moviliza el $55 \%$ del comercio exterior. No obstante, presenta un deterioro progresivo, con graves problemas de erosión y más del $50 \%$ de su territorio deforestado (Loaiza, et al., 2012).

9 Jovel, R. (2000): "El impacto del Fenómeno de El Niño de 1997-1998 en la Comunidad Andina de Naciones". Costa Rica: EIRD Informa - América Latina y el Caribe 1 (1). [Fecha de consulta: 10 marzo de 2013]. Disponible en: http://www.eird.org/esp/revista/Nol_2001/pa gina22.htm (Verificada: 27/08/15).

10 Corporación Autónoma Regional del Valle del Cauca - CVC. (2008): Ficha técnica cuenca Dagua. Santiago de Cali, Grupo Sistema de Información Ambiental, Dirección Técnica Ambiental. 


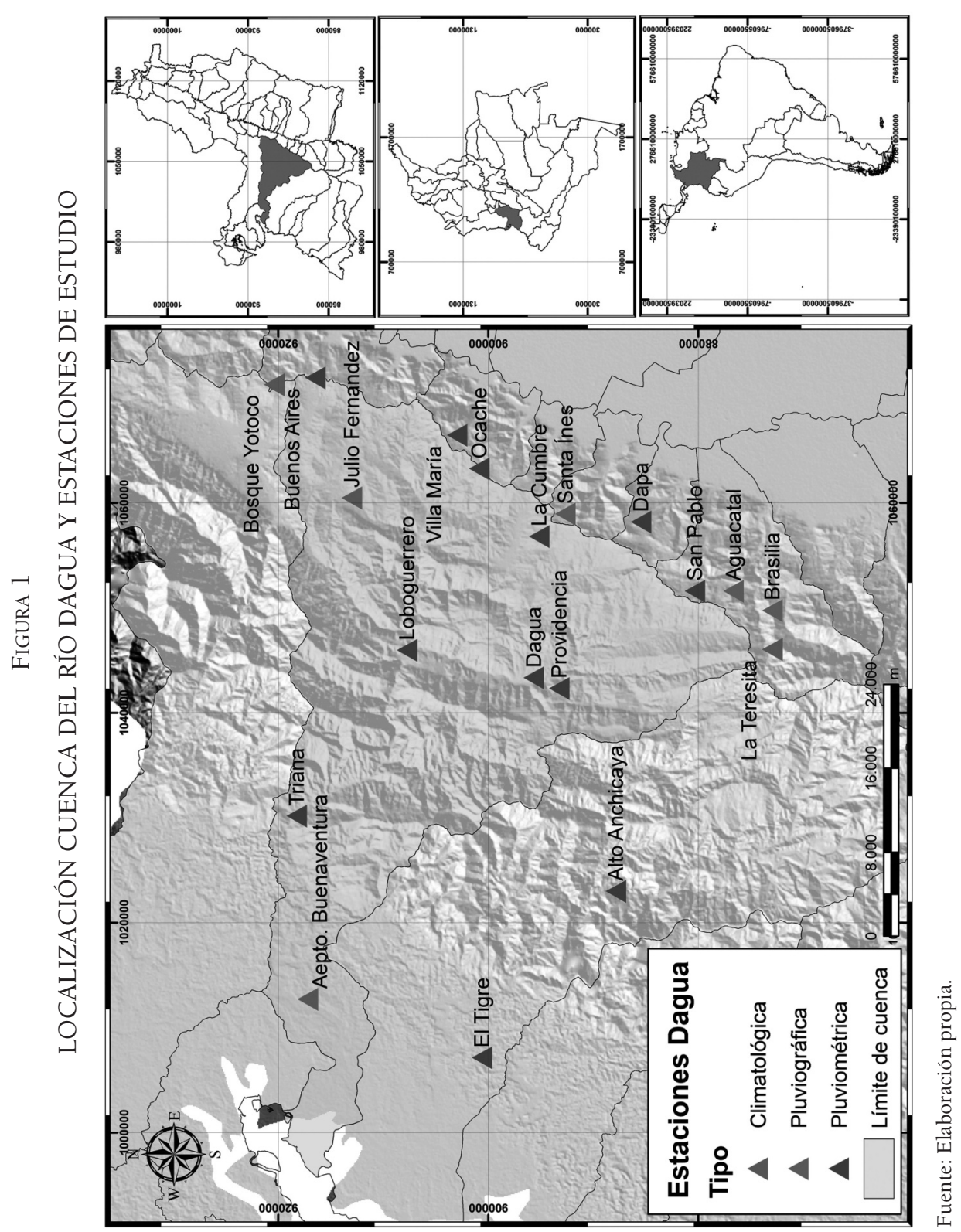

Estudios Geográficos, Vol. LXXVI, 279, pp. 557-578, julio-diciembre 2015

ISSN: 0014-1496, eISSN: 1988-8546, doi: 10.3989/estgeogr.201520 
Se preseleccionaron los registros mensuales de 23 estaciones ubicadas en la cuenca del río Dagua y sus alrededores. Posteriormente, mediante criterios de selección tales como: cobertura de la zona de interés, tiempo de registro mayor a 25 años y porcentaje máximo de datos faltantes del 10\%; se escogieron 19 estaciones cuya localización se observa en la figura 1.

\section{Metodología}

Para el desarrollo metodológico del SPI se recopilaron estudios realizados en la zona, y diferentes tipos de información, como la espacial proveniente de bases cartográficas, hasta la temporal que incluye datos pluviométricos, estudios técnicos, etc., así como los últimos avances del tema a nivel mundial.

El Índice de Precipitación Estandarizada, SPI cuantifica el déficit de precipitación para una gran variedad de escalas de tiempo lo que lo hace apto para estudiar sequías de diferentes duraciones, desde un mes (relevantes para la agricultura) a varios meses (relevantes para evaluar la disponibilidad de agua). El SPI es calculado ajustando la distribución de frecuencia de la precipitación con una función teórica de densidad de probabilidad (Mckee et al., 1993). La función de densidad es luego transformada a una distribución normal estandarizada (con media 0 y varianza 1), siendo el SPI el resultado de esta transformación (Nuñez et al., 2005).

La versatilidad temporal del índice es útil para determinar el comienzo y el fin de eventos secos que es una situación difícil de reconocer por medio de otros índices; además permite el análisis de los impactos de las sequías a distintas escalas temporales (Loaiza, et al., 2014; Vicente \& López, 2005; Vicente et al., 2011; Vincent, 2007; Pasho et al., 2011). A nivel mundial, de acuerdo con datos del NDMC, alrededor de 70 países utilizan el SPI para monitorear y analizar las sequías (Morales, 2005). Así, el SPI representa el número de desviaciones estándar que cada dato de precipitación se desvía del promedio histórico. Mckee et al. (1995) plantearon una clasificación de la sequía de acuerdo al SPI. 
TABLA 1

CLASIFICACIÓN DEL SPI

\begin{tabular}{l|l|l}
\hline \multicolumn{1}{c|}{ SPI } & \multicolumn{1}{c|}{ Categoría } & Gama de colores \\
\hline Mayor a 2,0 & Humedad extrema & \\
2,0 a 1,5 & Humedad severa & \\
1,5 a 1,0 & Cerca de lo normal & \\
1,0 a $-1,0$ & Sequía suave & \\
$-1,0$ a $-1,5$ & Sequía severa & \\
$-1,5$ a $-2,0$ & Sequía extrema & \\
Menor a $-2,0$ & & \\
\hline
\end{tabular}

Fuente: Adaptado de Mckee et al. (1995).

La magnitud es un solo valor de signo positivo correspondiente a la suma de los SPI durante el evento seco que inicia cuando el SPI es $\leq-1$ y termina cuando el SPI $\geq-1$. La «intensidad» de la sequía viene determinada por el valor promedio que el SPI alcanza dentro del período seco. Para obtener la intensidad, se calculó el valor promedio de las intensidades de todas las agrupaciones trimestrales de cada estación, y con apoyo del ArcGis se elaboró el mapa de intensidad media de la sequía meteorológica; de esta forma, se determinaron los eventos de sequía, magnitudes e intensidades.

Seguidamente, se realizó la frecuencia de ocurrencia de eventos de sequía para diferentes magnitudes, para lo cual, se analizó el riesgo de ocurrencia de diferentes magnitudes para la agrupación trimestral según la clasificación utilizada por Ángel \& Martelo (2008): leve (1 a 2), poco fuerte (2 a 3), fuerte (3 a 4), muy fuerte ( 4 a 5 ) y extremadamente fuerte ( $\geq 5$ ). Posteriormente se elaboraron los mapas de distribución espacial de la frecuencia de ocurrencia de diferentes tipos de sequías para cada magnitud.

Para la cobertura espacial de la sequía, se calculó el porcentaje de estaciones afectadas por valores de SPI $\leq-1$. Con los resultados, se seleccionaron un grupo de trimestres que presentan sequía en una alta proporción de estaciones, para los cuales se detalló a través de mapas, la distribución espacial de la intensidad. 


\section{RESULTADOS Y DISCUSIÓN}

A continuación se presentan los resultados del SPI para la agrupación trimestral, por ser la que mejor refleja los indicadores de impacto de la sequía en la agricultura de secano (maíz, fríjol, habichuela, etc.). Los resultados generales indican que la intensidad de las sequías no varía mucho en la zona de estudio, las estaciones presentan valores de sequías severas (SPI entre -1,5 y -2) y por tanto el mapa de intensidades no muestra tendencias espaciales significativas.

Al observar la figura 2, los períodos negativos del SPI en la estación Providencia coinciden con los años clasificados como El Niño, en los cuales la NOAA indica sobre la intensidad fuerte del fenómeno: 1982-83, 1991-92 y 1997-98, de los cuales el evento de 1991-92 presentó la mayor magnitud de sequía en toda la región; además de ciclos de intensidad moderada como son 1987-88, 1994-95 y 2006-07. Aunque el índice oceánico de El Niño (ONI por sus siglas en inglés) califica al evento 2009-10 como fuerte según el estándar utilizado, el Índice Multivariado del ENSO (MEI por sus siglas en inglés) y el Índice de Oscilación del Sur SOI (por sus siglas en inglés), lo determinan como de intensidad moderada; por lo que en conjunto se estableció que la intensidad del fenómeno fue moderada.

\section{FIGURA 2}

TRANSCURSO DEL SPI TRIMESTRAL ENTRE 1982 Y 2011 - ESTACIÓN PROVIDENCIA

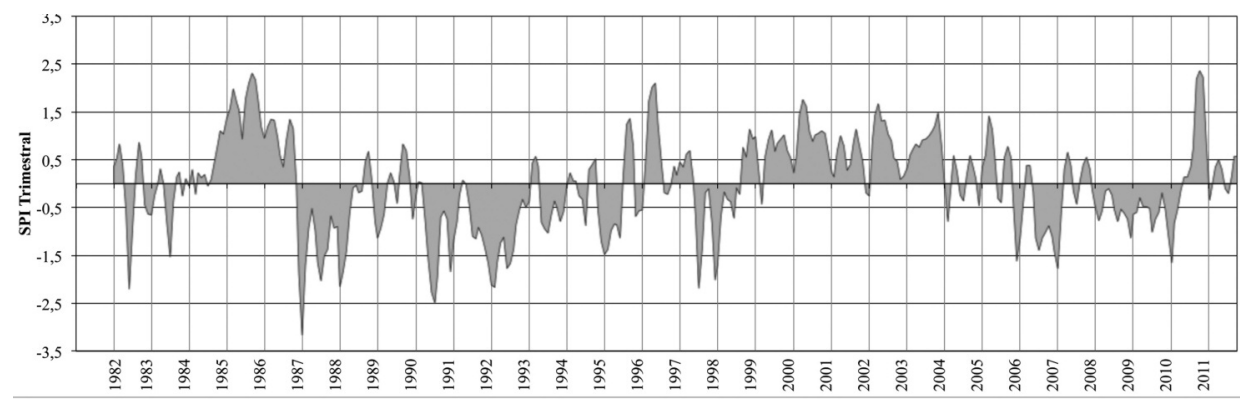

Fuente: Elaboración propia.

Los resultados indican un total de 545 eventos ( $8,2 \%$ del total de casos) de los 6612 posibles. Este bajo porcentaje explica por qué se ha asumido tradicionalmente que en el trópico húmedo (localización de Colombia) casi no 
ocurren sequías; sin embargo, es un valor que llama a revisar con especial cuidado porque en efecto, independientemente de la causa, el problema real es no contar con el agua necesaria para los procesos vitales de los cultivos, ecosistemas y actividades socioeconómicas.

En la tabla 2 se presentan las sequías leves con el 39,1\% del total de eventos, poco fuertes, con el 18,9\% de los casos y, el 16\% de las sequías extremadamente fuertes. En la tabla 3 se presentan las 10 magnitudes máximas de las sequías detectadas en la zona de estudio. Para caracterizar la distribución del patrón espacial de las diferentes magnitudes de sequía, se presentan los mapas que reflejan su frecuencia de ocurrencia; la figura 3 presenta el resultado para la sequía extremadamente fuerte.

TABLA 2

FRECUENCIA DE OCURRENCIA (\%) DE DIFERENTES TIPOS DE SEQUÍA

\begin{tabular}{lrrrrrr}
\hline \multirow{2}{*}{ Magnitud de la sequía } & Leve & $\begin{array}{c}\text { Poco } \\
\text { fuerte }\end{array}$ & Fuerte & $\begin{array}{c}\text { Muy } \\
\text { fuerte }\end{array}$ & $\begin{array}{c}\text { Extremadamente } \\
\text { Fuerte }>=5\end{array}$ & TOTAL \\
\cline { 2 - 5 } & 1 a 2 & 2 a 3 & 3 a 4 & 4 a 5 & 87 & 545 \\
\hline Número de eventos & 213 & 103 & 80 & 62 & 16 & 100 \\
Porcentaje (\%) & 39,1 & 18,9 & 14,7 & 11,4 & \\
\hline
\end{tabular}

Fuente: Elaboración propia.

TABLA 3

MAGNITUDES MÁXIMAS DE LAS SEQUÍAS DETECTADAS EN LA CUENCA DAGUA

\begin{tabular}{lcclcl}
\hline \multicolumn{1}{c}{ Estación } & Magnitud & $\begin{array}{c}\text { Duración } \\
\text { (meses })\end{array}$ & Estación & Magnitud & $\begin{array}{c}\text { Duración } \\
\text { (meses) }\end{array}$ \\
\hline San Pablo & 32,94 & Dic 86 - May 88 (18) & Anchicayá & 10,55 & Ene 92 - Sep 92 (9) \\
Providencia & 17,09 & Oct 91 - Oct 92 (13) & El Tigre & 9,33 & Oct 00 - Abr 01 (7) \\
Triana & 14,28 & May 94 - Feb 95 (10) & Brasilia & 9,02 & Abr 90 - Sep 90 (6) \\
Dapa & 12,12 & Dic 84 - Ago 85 (9) & $\begin{array}{l}\text { Julio Fernán- } \\
\text { dez }\end{array}$ & 8,71 & Oct 91 - Mar 92 (6) \\
Aguacatal & 11,54 & Abr 09 - Nov 09 (8) & Dagua & 8,35 & Dic 91 - Jun 92 (7) \\
\hline
\end{tabular}

Fuente: Elaboración propia.

Estudios Geográficos, Vol. LXXVI, 279, pp. 557-578, julio-diciembre 2015

ISSN: 0014-1496, eISSN: 1988-8546, doi: 10.3989/estgeogr.201520 


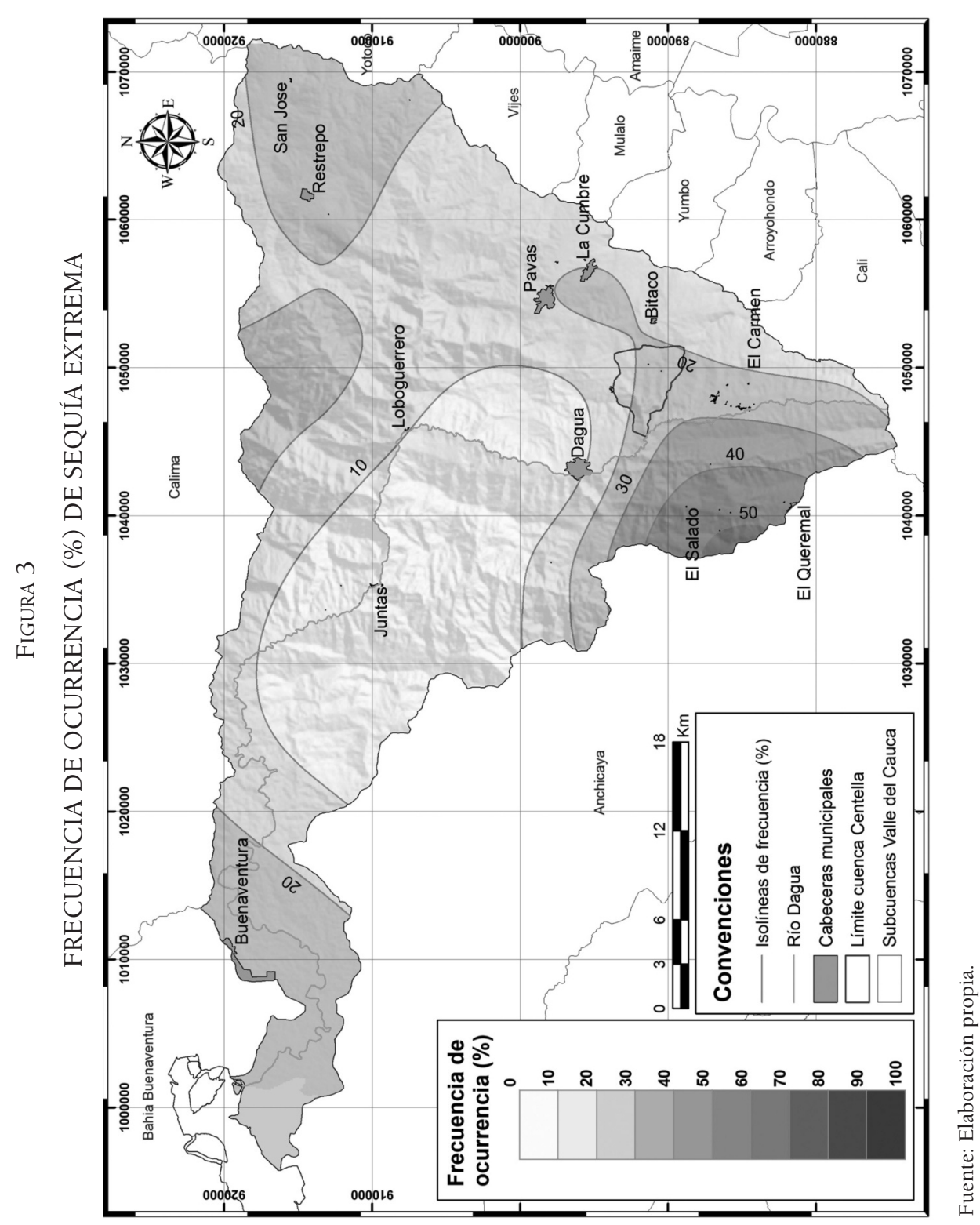

Estudios Geográficos, Vol. LXXVI, 279, pp. 557-578, julio-diciembre 2015 ISSN: 0014-1496, eISSN: 1988-8546, doi: 10.3989/estgeogr.201520 
Se registraron 87 sequías extremadamente fuertes; su distribución espacial muestra una fuerte concentración de frecuencias superiores al 50\% en la parte alta de la cuenca (ver figura 3). La zona que predomina corresponde a las frecuencias de ocurrencia entre el $10 \%$ y el $20 \%$, la cual cubre toda la parte baja y media de la cuenca. Las sequías de magnitudes muy fuerte y extremadamente fuerte están asociadas a duraciones de varias agrupaciones temporales consecutivas de intensidades severas y extremas. Los resultados indican que ocurrieron 62 eventos y que espacialmente la zona alta y el centro de la cuenca, presentan la mayor frecuencia de ocurrencia (30\% a $40 \%$ de los casos). En el período de registro ocurrieron 80 sequías de magnitud fuerte; la distribución espacial de la frecuencia de ocurrencia de estos eventos presenta una concentración hacia la parte media de la cuenca con un porcentaje entre $30 \%$ y el $40 \%$, que cubre la cabecera municipal de Dagua.

Las sequías de magnitud poco fuerte y fuerte están asociadas a duraciones de varias agrupaciones temporales consecutivas pero con intensidades bajas. En el período de registro ocurrieron 103 sequías de magnitud poco fuerte; las principales poblaciones se encuentran en las zonas de menor frecuencia de ocurrencia (0 a 20\%). En general las sequías leves están asociadas a duraciones de una agrupación temporal, aunque pueden tener una intensidad alta. Por ejemplo, para el trimestre de septiembre de 1986 el SPI fue de -2,12 (Extremadamente seco), pero esta situación duró solo un trimestre. En el período de registro ocurrieron 213 eventos de esta magnitud. Las frecuencias más altas (50\% a 60\%) ocurren en la parte media de la cuenca sobre el bioma de selva sub-andina y el enclave subxerofítico del río Dagua.

Con el objetivo de encontrar la lámina de lluvia $(\mathrm{mm})$ que corresponde al límite mínimo para que ocurra una sequía, se retrotrajeron los valores del SPI = -1 en cada estación de análisis; de tal modo que al obtener las láminas de lluvia que corresponden a dicho SPI, se obtiene el valor umbral de precipitación para el inicio de la sequía; por tanto, si en una agrupación trimestral «X» no logra acumularse esa cantidad de lluvia, en dicha agrupación ocurriría una sequía.

En general, los acumulados de lluvia trimestrales por debajo de los cuales se produce una sequía tienden a presentar los valores más pequeños hacia la parte media de la cuenca, oscilando en un rango entre 0 y 150 mm; así mismo, aumentan hacia el oeste en la parte baja de la cuenca, la cual presenta un régimen de lluvias monomodal en el cual predominan precipitaciones durante todo el año que alcanza un umbral promedio de $1200 \mathrm{~mm}$ trimestrales. En cuanto a la parte alta, los umbrales de precipitación se encuentran entre los $300 \mathrm{~mm}$ trimestrales y disminuyen a medida que llegan al piedemonte de la cordillera. 
Para analizar las intensidades de la sequía en la cuenca Dagua se eligieron algunos eventos particulares con base a los niveles de cobertura espacial. Los casos se tomaron de aquellas sequías que afectaron a un gran número de estaciones en el área de estudio. Se utilizó como proxy el porcentaje de estaciones afectadas por sequía (SPI $\leq-1$ ) calculado para todas las agrupaciones trimestrales del registro histórico (ver figura 4).

Los resultados de cobertura indican que de los 358 trimestres analizados, el 40,8\% no registró eventos de sequía en ninguna de las estaciones estudiadas; 34 afectaron más del $60 \%$ de las estaciones presentes en la zona de estudio, y los trimestres que afectaron más del $75 \%$ de las estaciones, los cuales coinciden con la clasificación de meses Niño de la NOAA, fueron: ago/1982, ago y sep/1983, feb/1987, ago/1990, feb, mar y ago/1991, feb/1995, ago y sep/1997, y feb/1998, como se muestra en la figura 4. Con base en esta información, se escogieron tres ejemplos de agrupaciones trimestrales para detallar espacialmente la intensidad y las zonas más afectadas por las sequías; estos fueron los casos extremos de Jun-Ago del 82, Dic del 90 a Feb del 91, Dic del 97 a Feb del 98. La figura 5 muestra el comportamiento espacial del SPI durante los trimestres mencionados, indicando una fuerte concentración de condiciones extremadamente secas hacia la parte alta y media de la cuenca.

Lo anterior se sustenta en la importancia de la ZCIT como el mecanismo físico de mayor relevancia en la explicación del ciclo anual de la hidroclimatología de Colombia sobre el centro y el occidente, dada la existencia de dos temporadas lluviosas (abril-mayo y octubre-noviembre) y dos temporadas secas (diciembre-febrero y junio-agosto) como resultado de la doble migración sobre el país (Mejía et al., 1999; León et al., 2000; Poveda 2004; Poveda et al., 2005), y de su interacción con las circulaciones de los océanos Pacífico, Atlántico y de la cuenca del Amazonas. Las dos épocas de reducción de lluvias coinciden con las más secas del año; situación que se intensifica cuando ocurre la fase cálida del fenómeno ENOS debido a varios factores que son objeto de estudio y que Poveda (2004), resume así: debilitamiento del chorro del Chocó, debilitamiento de la corriente de chorro ecuatorial (700 hPa.), reducción en la intensidad y número de ondas tropicales del este sobre el Atlántico tropical norte, desplazamiento de la ZCIT hacia el suroeste de su posición normal como consecuencia de una celda de Hadley anómala sobre el trópico americano, y la alteración del balance de humedad atmosférica (Cuartas \& Poveda, 2002).

El análisis de coincidencia gráfica entre los eventos extremos del ENOS (Representados en el ONI) y los picos negativos y positivos del SPI, que muestran periodos secos y lluviosos, se verificó con las correlaciones obtenidas de 


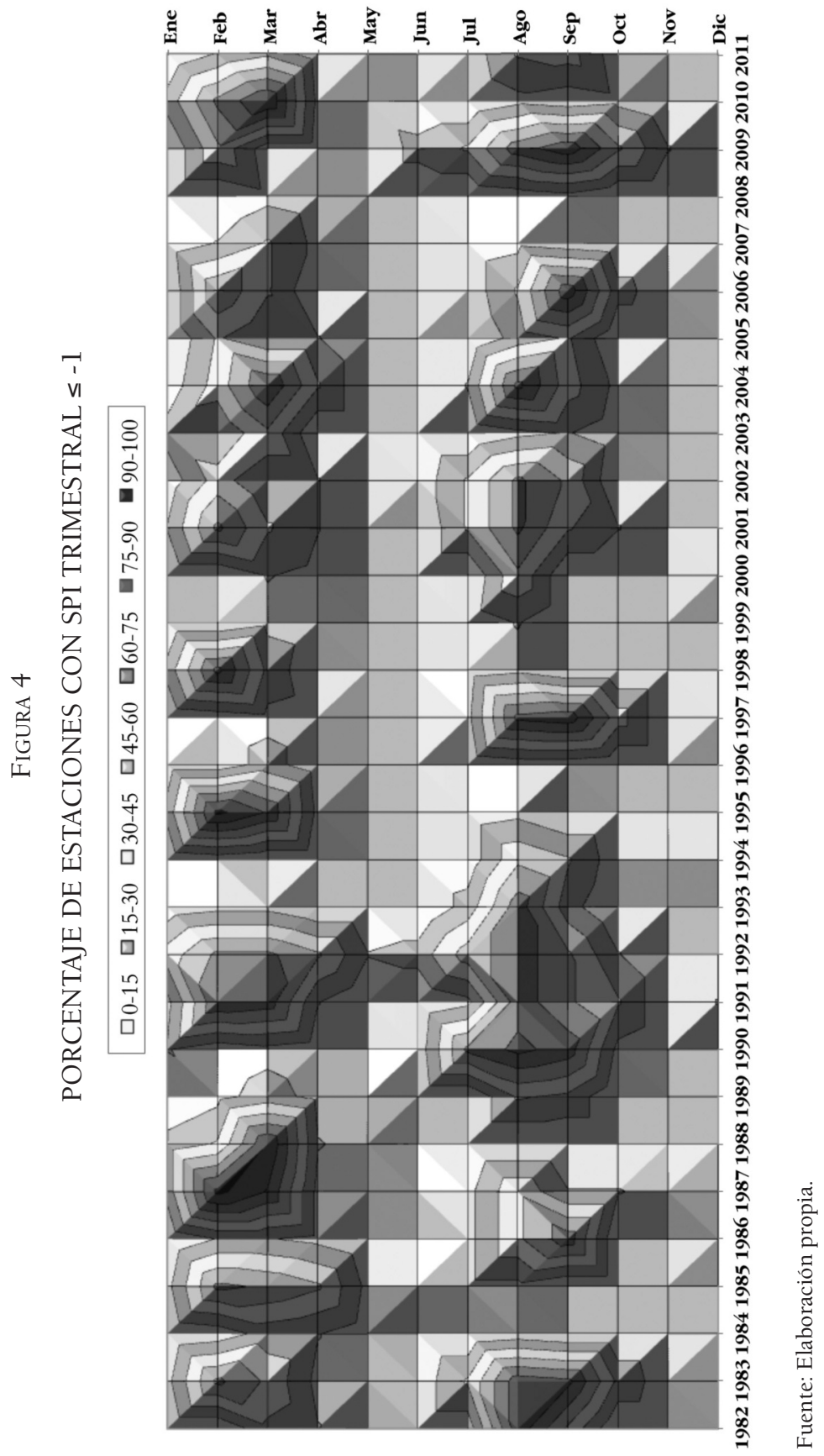

Estudios Geográficos, Vol. LXXVI, 279, pp. 557-578, julio-diciembre 2015

ISSN: 0014-1496, eISSN: 1988-8546, doi: 10.3989/estgeogr.201520 


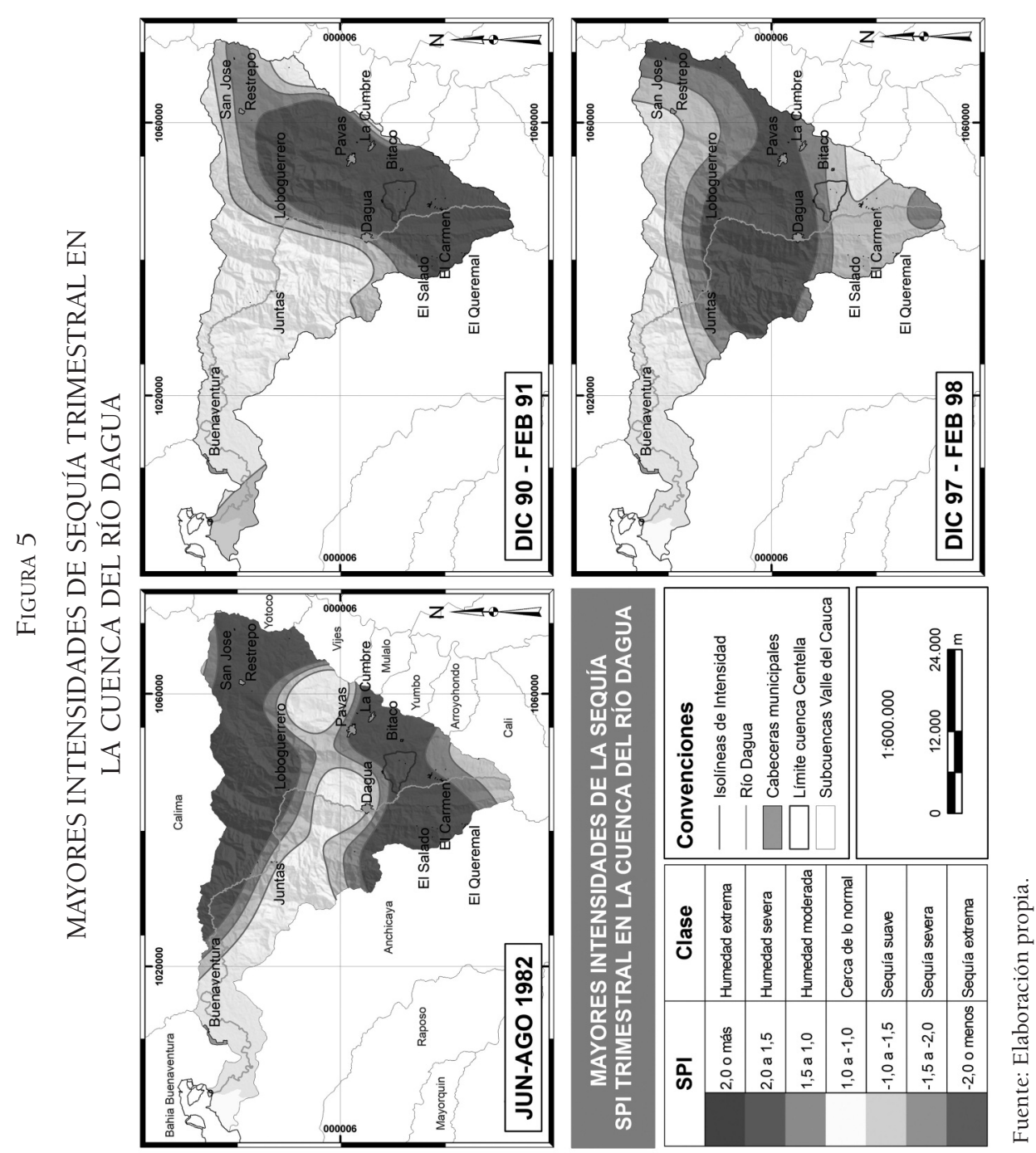


asociar la serie trimestral las series del ONI con el SPI trimestral; el ONI es un índice que relaciona las temperaturas superficiales del Océano Pacifico en la región conocida como Niño 3-4, que es la que tiene mayor asociación con la climatología Colombiana, según estudios precedentes (Poveda et al., 2010; Mesa, 2008; Carvajal, 2004); las correlaciones significativas del ONI con el SPI, alcanzaron valores entre - 0,28 y - 0,40 en las estaciones; el signo negativo indica que la temperatura del Océano en ésta región del Pacífico es inversamente proporcional al valor del SPI. Similares resultados, se obtuvieron, correlacionando las series del MEI con el SPI, índice basado en 6 variables principales sobre el Océano Pacífico Tropical, que monitorean el comportamiento del ENOS; estas 6 variables son presión al nivel del océano, componentes zonales y meridionales del viento superficial, temperatura superficial del océano, temperatura del aire superficial, y la fracción total de nubosidad del cielo, (Wolter, 1987).

Durante el período junio-agosto de 1982 predominaron las sequías extremas en el 45\% de la zona, principalmente en la parte alta y hacia el centronorte de la cuenca; y prevalecieron las condiciones normales en la parte baja que cubrieron el $28 \%$ del área. En cuanto a las sequías suaves y extremas, cada una representó el 13\% de las condiciones secas en el territorio.

Para el trimestre diciembre del 90 a febrero del 91 las condiciones secas se concentraron en la parte alta de la cuenca, las sequías extremas cubrieron el $35 \%$, las severas el $10 \%$ y las suaves el $13,5 \%$, mientras que en la parte media y baja imperaron condiciones cercanas a lo normal y en menor proporción sequías suaves. Finalmente, para el trimestre de febrero del 98, las sequías extremas ocuparon $30 \%$ de la cuenca principalmente en la zona media, hacia la zona baja se mantienen las condiciones normales y en la microcuenca Centella prevalecieron las sequías severas y suaves, las cuales ocuparon el 19\% y $22 \%$ del territorio respectivamente.

\section{CONCLUSIONES}

El Índice Estandarizado de Precipitación aplicado a la cuenca del río Dagua demostró ser un indicador adecuado de la sequía meteorológica, mediante el cual se reconocieron los eventos de sequía para diferentes agrupaciones temporales (mes, trimestre y semestre), los cuales coincidieron con los años clasificados como El Niño, en los cuales la NOAA indica sobre la intensidad fuerte del fenómeno, siendo 1991-92 el de mayor magnitud de sequía en la región. El SPI también permitió clasificar los eventos de sequía para la agrupación trimestral 
según cinco aspectos: intensidad, duración, magnitud, cobertura espacial y umbrales de precipitación; se logró diferenciar espacialmente la gravedad de los eventos de sequía, la concentración de los mismos y los valores de lámina de lluvia que indican sequía. En promedio, en la zona ocurren dos sequías leves de al menos un mes de duración, una poco fuerte, una fuerte y una sequía extrema cada diez años, afectando al menos una estación meteorológica, lo que implica que la agricultura y en general la biodiversidad local son muy susceptibles.

El SPI constituye uno de los elementos básicos para desarrollar una planificación estratégica, que permita la toma de decisiones requeridas a mediano y largo plazo para enfrentar de manera eficiente los diferentes eventos de sequía que podrían presentarse; a través del desarrollo e implantación de sistemas de producción menos vulnerables (prevención), y la reducción de los impactos negativos (mitigación). Es claro que se requerirá de información socioeconómica para determinar la vulnerabilidad y poder tomar decisiones correctas, pero disponer de los mapas es un paso fundamental para lograrlo.

Debido a la complejidad del fenómeno es recomendable fragmentar su análisis de acuerdo con la disponibilidad de información y las necesidades particulares del contexto en el que se trabaje. En muchos casos el uso de índices de sequía se reduce a aquellos que es posible aplicar con información escasa, principalmente los que solo necesitan el uso de la precipitación para su cálculo. Algunos índices como los de balance hídrico encuentran problemas importantes en la disponibilidad de información y los costos de su recolección. El Índice de Palmer es un ejemplo importante. A pesar de esto, se han desarrollado índices pensados para suplir las necesidades de algunas regiones, diseñados de acuerdo a las características locales, cuya implementación se reduce a pequeñas áreas que restringen el uso en otros países. El uso del SPI para Dagua, aunque vislumbra algunas características importantes de la sequía, no da lugar a generalizaciones en la definición del fenómeno y de sus eventos; es un análisis en construcción continua, y se considera un punto de partida que deberá apuntar a un estudio en el que se cruce información sobre la oferta natural y la demanda de agua.

Es recomendable realizar una zonificación de la vulnerabilidad a sequías en la cuenca del río Dagua, usando información socioeconómica y ambiental, teniendo en cuenta diferentes criterios: zonas productoras de agua, centros poblados, zonas forestales en las que podrían incrementarse los incendios, zonas agrícolas, embalses, zonas de producción ganadera, entre otras, así como establecer los indicadores para clasificar los rangos de vulnerabilidad a la sequía de los sectores social, hídrico y agrícola. Se requieren estudios más detallados que correlacionen la valoración económica de los impactos socioeconómicos con 
las diferentes magnitudes e intensidades de la sequía para diferentes agrupaciones temporales, que a su vez permitan una adecuada zonificación de la vulnerabilidad ante la sequía meteorológica en la cuenca del río Dagua. Estos resultados, crearan las condiciones organizacionales, de infraestructura y económicas, que permitan dar respuesta eficiente y eficaz ante un evento de sequía, contribuyendo a mitigar al menos en parte, sus efectos negativos.

Fecha de recepción: 14 de julio de 2014.

Fecha de aceptación: 26 de febrero de 2015.

\section{BIBLIOGRAFÍA}

Aldunce, P.; Carvajal, Y.; León, A.; Neri, C.; Quintero, M. \& Soza, S. (2009): Sistematización de las políticas y estrategias de adaptación nacional e internacional al cambio climático del sector silvoagropecuario y de los recursos hídricos y edáficos. Santiago de Chile, Dpto Ciencias Ambientales y Recursos Naturales, Facultad de Ciencias Agr., Universidad de Chile, 132 p.

Ángel, R. \& Martelo, M. (2008): "Caracterización de la sequía meteorológica en los climas árido, semiárido y subhúmedo seco en los Llanos Centro Orientales de Venezuela". (Magister en Gerencia Ambiental). Caracas, Universidad Nacional Experimental Politécnica de la Fuerza Armada \& Centro de Investigación y Postgrado UNEFA-CIP, 216 pp.

Below, R.; Grover-Kopec, E. \& Dilley, M. (2007): "Documenting Drought-Related Disasters: A Global Reassessment". The Journal of Environment \& Development, 16, pp. 328-344.

Brown, M. \& Funk, C. (2008): "Climate - Food security under climate change". NASA Publications. [en línea]. [Fecha de consulta: 10 de febrero de 2014]. Disponible en: http://digitalcommons.unl.edu/cgi/viewcontent.cgi?article=1130\&context=nasapub (Verificada: 26/08/2015).

Carvajal, Y. (2004): "El uso de funciones ortogonales empíricas y análisis de correlación canónica en el estudio de la variabilidad hidrometeorológica, aplicación al Valle del Cauca-Colombia". Tesis (Doctorado en Hidráulica y Medio Ambiente), Valencia, Universidad Politécnica de Valencia, 461 pp.

Carvajal, Y.; Quintero, M. \& Garcia, M. (2008): "Women's role in adapting to climate change and variability". Advances in Geosciences. [en línea]. 14, pp. 277-280. [Fecha de consulta: 10 de febrero de 2014]. Disponible en: http://www.advgeosci.net/14/277/2008/adgeo-14-277-2008.pdf (Verificada: 26/08/2015).

Cuartas, A. \& Poveda, G. (2002): "Balance atmosférico de humedad y estimación de la precipitación reciclada en Colombia según el Reanálisis NCEP/NCAR". Meteorología Colombiana, [en línea], 5, pp. 49-57. [Fecha de consulta: 12 de diciembre de 2013]. 
Disponible en: http://ciencias.bogota.unal.edu.co/fileadmin/content/geociencias/ revista_meteorologia_colombiana/numero05/05_06.pdf (Verificada: 27/08/15).

Debels, P.; Szlafsztein, C.; Aldunce, P.; Neri, C.; Carvajal, Y.; Celis, A.; Bezanilla, A. \& Martínez, D. (2008): "IUPA: a proposal of an index for the evaluation of the general usefulness of practices for adaptation to climate change and variability". Natural Hazards, 50, pp. 211-233.

Guttman, N. (1998): "Comparing the Palmer drought index and the Standardized Precipitation Index". Journal of the American Water Resources Association, [en línea], 34/1. [Fecha de consulta: 9 de marzo de 2013]. Disponible en: http:// onlinelibrary.wiley.com/doi/10.1111/j.1752-1688.1998.tb05964.x/pdf (Verificada: 27/08/15).

Kallis, G. (2008): "Droughts". Annual Review of Environment and Resources, 33, pp. 85-118.

Lavell, A.; Mansilla, E. \& Smith, D. (2003): "La gestión local del riesgo: nociones y precisiones en torno al concepto y la práctica”. [en línea], Managua, CEPREDENAC - PNUD. [Fecha de consulta, 14 de febrero de 2014]. Disponible en: http://www.cridlac.org/digitalizacion/pdf/spa/doc15783/doc15783-contenido.pdf (Verificada: 27/08/15).

León, G.; Zea, J. \& Eslava J. (2000): "Circulación general del trópico y la zona de confluencia intertropical en Colombia”. Meteorología Colombiana, [en línea], 1, pp. 31-38. [Fecha de consulta: 10 de enero de 2014]. Disponible en: Circulación general del trópico y la zona de confluencia intertropical en Colombia. (Verificada: 27/08/15).

Loaiza, W.; Carvajal, Y. \& Baquero, O. (2014): Sequías y Adaptación. Principios para su evaluación en sistemas productivos agrícolas del Valle del Cauca, Colombia. Cali, Programa editorial de la Universidad del Valle, $149 \mathrm{pp}$.

Loaiza, W.; Reyes, A. \& Carvajal, Y. (2012): "Aplicación del ISRHA para definir estrategias tecnológicas sostenibles en la microcuenca Centella”. Revista Ingeniería y Desarrollo, 30/2, pp. 160-181.

Marcos Valiente, O. (2001): "Sequía: definiciones, tipologías y métodos de cuantificación”. Investigaciones Geográficas, 26, pp. 59-80.

Mckee, T.; Doesken, N. \& Kleist, J. (1995): "Drought monitoring with multiple time scales", en: Proceeding of the Ninth Conference on Applied Climatology [January 1520, 1995, Dallas USA. TX]. Dallas, American Meteorological Society, pp. 233-236.

Mckee, T.; Doesken, N. \& Kleist, J. (1993): "The relation of drought frequency and duration to time scales", en: Proceeding of the Eight Conference on Applied Climatology [January 17-22, 1993, Boston. USA]. Boston, American Meteorological Society, pp. 179-184.

Mejía, F; Mesa, O.; Poveda, G. et al. (1999): "Distribución espacial y ciclos anual y semianual de la precipitación en Colombia”. DYNA, 127/66, pp. 7-15.

Mendelsohn R. \& Dinar A. (1999): "Climate Change, Agriculture, and Developing Countries: Does Adaptation Matter?" World Bank Research Observer, [en línea], 14, 
pp. 277-293. [Fecha de consulta: 17 de febrero de 2014]. Disponible en: http://wbro.oxfordjournals.org/content/14/2/277.abstract (Verificada: 27/08/15).

Mesa, O. (2008): “¿A dónde va a caer este Globo? Acerca del futuro de la Tierra”. Medellín, Universidad Nacional de Colombia, 328 p.

Morales, J. (2005): "El efecto de la longitud de registro en el cálculo del índice de precipitación estándar" (Maestría en Ingeniería Hidráulica), México, Universidad Nacional Autónoma de México, Programa de Maestría y Doctorado en Ingeniería, $111 \mathrm{pp}$.

National Drought Mitigation Center - NDMC. (2006): What is Drought? National Weather. [Fecha de consulta: 10 marzo de 2013]. Disponible en: http://www.nws. noaa.gov/om/brochures/climate/Drought.pdf (Verificada: 28/08/2015).

Pasho, E.; Camarero, J.; De Luis, M. \& Vicente, S. (2011): "Impacts of drought at different time scales on forest growth across a wide climatic gradient in north-eastern Spain". Agricultural and Forest Meteorology, [en línea], 151, pp. 1800-1811. [Fecha de consulta: 16 de febrero de 2014]. Disponible en: http://www.sciencedirect.com/ science/article/pii/S016819231100253X (Verificada: 27/08/15).

Patrick, E. (2003): Sequía: Vulnerabilidad y crisis en tierras áridas, Kenia, Centro para el Desarrollo de las Tierras Áridas, Programa de las Naciones Unidas para el Desarrollo (PNUD), 2 pp.

Poveda, G. (2004): "La Hidroclimatología de Colombia: Una síntesis desde la escala inter-decadal hasta la escala diurna". Revista Academia Colombiana de Ciencias, [en línea], 28/107, pp. 201-222. [Fecha de consulta: 15 de febrero de 2014]. Disponible en: http://users.clas.ufl.edu/prwaylen/geo3280articles/Synthesis\%20of\%20Colombian\%20hydrology.pdf (Verificada: 27/08/15).

Poveda, G.; Alvarez, D. \& Rueda, O. (2010): "Hydroclimatic variability over the Andes of Colombia associated with enso: a review of climatic processes and their impact on one of the Earth's most important biodiversity hotspots". Climate Dynamics, [en línea], 36, pp. 2233-2249. [Fecha de consulta 10 de febrero de 2014]. Disponible en: http://link.springer.com/article/10.1007\%2Fs00382-0100931-y (Verificada: 27/08/15).

Poveda, G.; Vélez, J.; Mesa, J. et al. (2005): "Linking long-term water balances and statistical scaling to estimate river flows along the drainage network of Colombia". Journal of Hydrologic Engineering, [en línea], 12 (1). [Fecha de consulta: 11 de enero de 2014]. Disponible en: http://www.clas.ufl.edu/users/prwaylen/geo3280articles/ Water\%20balance\%20calculations\%20for\%20Colombial.pdf (Verificada: 27/08/15).

Rahman, A. \& Westley, J. (2002): "The Challenge of Ending Rural Poverty". Development Policy Review, [en línea], 19/4, pp. 553-562. [Fecha de consulta: 10 de diciembre de 2013]. Disponible en: http://onlinelibrary.wiley.com/doi/10.1111/14677679.00152/pdf (Verificada: 27/08/15).

Rojo, J. \& Carvajal, L. (2010): "Predicción no lineal de caudales utilizando variables macroclimáticas y análisis espectral singular". Revista Tecnología y Ciencias del Agua, antes Ingeniería hidráulica en México, México, 1/4, pp. 59-73. 
Seth, S. (2003): "Chapter 8. Human impacts and management issues in arid and semiarid regions". Simmers, I. Understanding water in a Dry Environment. Hydrological processes in arid and semiarid 577 zones, IAH International Contributions to Hydrogeology, 23, pp. 289-342.

Vargas, J. (2002): "Políticas públicas para la reducción de la vulnerabilidad frente a los desastres naturales y socio-naturales". Serie medio ambiente y desarrollo CEPAL, [en línea], 79. [Fecha de consulta: 15 febrero de 2014]. Disponible en: http://www. eclac.org/publicaciones/xml/1/10561/lcl1723e_1.pdf (Verificada: 27/08/15).

Vincent, K. (2007): "Uncertainty in adaptive capacity and the importance of scale". Global Environmental Change, [en línea], 17, pp. 12-24. [Fecha de consulta: 15 noviembre de 2013]. Disponible en: http://www.sciencedirect.com/science/article/pii/ S0959378006000884 (Verificada: 27/08/15).

Vicente, S.; Beguería, S. \& López, J. (2011): "Comment on "Characteristics and trends in various forms of the Palmer Drought Severity Index (PDSI) during 1900-2008". Journal of Geophysical Research, [en línea], 116, pp. 1-9. [Fecha de consulta: 17 de febrero de 2014]. Disponible en: http://digital.csic.es/bitstream/10261/41161/1/BegueriaS_JGeophysRes_2011.pdf (Verificada: 27/08/15).

Vicente, S. \& López, J. (2005): "Hydrological response to different time scales of climatological drought: an evaluation of the Standardized Precipitation Index in a mountainous Mediterranean basin". Hydrology and Earth System Sciences, [en línea], 9, pp. 523-533. [Fecha de consulta: 25 de agosto de 2013]. Disponible en: http://www.hydrol-earth-syst-sci.net/9/523/2005/hess-9-523-2005.pdf (Verificada: 27/08/15).

Wilhite, D. \& Buchanan, M. (2005): "Drought as Hazard: Understanding the Natural and Social Context", en D. Drought Wilhite and Water Crises: Science, Technology, and Management Issues, Boca Raton, FLCRC Press, pp. 3-29.

Wolter, K. (1987): "The Southern Oscillation in surface circulation and climate over the tropical Atlantic, Eastern Pacific, and Indian Oceans as captured by cluster analysis". J. Climate Appl. Meteor., 26, pp. 540-558.

Zuluaga, J. (2009): Análisis de la variabilidad espacio - temporal de la sequía en Colombia (Maestría). Medellín, Universidad Nacional de Colombia - Sede Medellín, Facultad de Minas, Escuela de Geociencias y Medio Ambiente, 100 pp.

\section{RESUMEN}

El Índice Estandarizado de Precipitación (SPI) caracteriza las sequías meteorológicas de un lugar, mediante la cuantificación del déficit de precipitación en diferentes períodos de tiempo. Valores negativos del SPI representan sequías, mientras valores positivos indican periodos húmedos. Los resultados muestran que los períodos SPI negativos, coinciden con años clasificados como El Niño: 82-83, 91-92 y 97-98, de los cuales, el evento 91-92 presentó los mayores impactos en la región. En general, los resultados indican que las sequías más intensas en la cuenca del Dagua, se presentaron 
en los trimestres de Dic-Ene-Feb, Ene-Feb-Mar, Jun-Jul-Ago y Jul-Ago-Sep; estos resultados se espacializaron con técnicas geoestadísticas, y son insumo básico para formular estrategias de adaptación y garantizar la soberanía alimentaria de los pequeños agricultores frente a las sequías.

Palabras Claves: Índice estandarizado de precipitación; fenómeno El Niño; sequías; variabilidad climática.

\section{AbStract}

The Standardized Precipitation Index (SPI) characterizes meteorological droughts of a place, by quantifying the deficit of rainfall in different time periods. Negative SPI values represents drought, while positive values indicate wet periods. The results suggest that negative SPI periods coincide with El Niño years: 82-83, 91-92 and 97-98. The 91-92 event had the largest impact on the region. These results indicate that the most severe droughts in the basin of Dagua, were presented at the quarters of DecJan-Feb, Jan-Feb-Mar, Jun-Jul-Aug and Jul-Aug-Sep. The results have been obtained with geostatistical techniques, and are a basic input to formulate adaptation strategies and ensure food sovereignty of small farmers against drought.

KEY WORDS: standardized precipitation index; El Niño; drought; climate variability.

\section{RÉSUMÉ}

L'Indice de précipitations normalisé (SPI) caractérise les sécheresses météorologiques d'un lieu, en quantifiant le déficit de précipitations à différentes périodes de temps. Les valeurs négatives de SPI indiquent périodes sécheresse, tandis que les positives indiquent des périodes humides. Les résultats montrent que les périodes SPI négatifs obtenus coïncident avec les années «El Niño». Les années en question sont: 82-83, 91-92 et 97-98. L'événement 91-92 montre le plus grand impact sur la région. Ces résultats suggèrent que les sécheresses les plus sévères touchant le bassin de Dagua ont eu lieu lors des trimestres de décembre-janvier-février, janvier-février-mars, juin-juillet-août et juin-août-septembre. Les résultats ont été obtenus à l'aide des techniques géostatistiques et fournissent des outils de base pour l'élaboration des stratégies d'adaptation afin d'assurer la souveraineté alimentaire des petits agriculteurs contre la sécheresse.

MoTS CLÉs: indice de précipitations normalisé; El Niño; sécheresse; la variabilité du climat. 